\title{
Evolution of Primordial Neutrino Helicities in Astrophysical Magnetic Fields and Implications for Their Detection
}

\author{
Gordon Baym® and Jen-Chieh Peng® \\ Illinois Center for Advanced Studies of the Universe and Department of Physics, University of Illinois, \\ 1110 West Green Street, Urbana, Illinois 61801, USA
}

(Received 13 December 2020; revised 13 April 2021; accepted 16 April 2021; published 12 May 2021)

\begin{abstract}
Since decoupling in the early Universe in helicity states, primordial neutrinos propagating in astrophysical magnetic fields precess and undergo helicity changes. In view of various experimental bounds allowing a large magnetic moment of neutrinos, we estimate the helicity flipping for relic neutrinos in both cosmic and galactic magnetic fields. The flipping probability is sensitive both to the neutrino magnetic moment and the structure of the magnetic fields and is a potential probe of the fields. As we find, even a magnetic moment well below that suggested by XENON1T could significantly affect relic neutrino helicities and their detection rate via inverse tritium beta decay.
\end{abstract}

DOI: 10.1103/PhysRevLett.126.191803

The early Universe was bathed with thermal neutrinos which decoupled from matter around $1 \mathrm{~s}$ after the big bang. Detection of these neutrinos, e.g., through inverse beta decay capture on tritium [1] in the Princeton Tritium Observatory for Light, Early-Universe, Massive-Neutrino Yield (PTOLEMY) experiment [2,3], remains a major challenge. Relic neutrinos carry information about the early Universe at a much earlier epoch than that of photon decoupling. In addition, neutrinos propagating through the Universe acquire information about the gravitational and magnetic fields they encounter en route to Earth. We focus here on the evolution of the helicity of primordial neutrinos and implications for their detection rates.

Neutrinos in the early Universe decoupled essentially in chirality eigenstates at temperatures orders of magnitude larger than neutrino masses, leaving these highly relativistic neutrinos essentially in helicity eigenstates as well. Were neutrinos to travel freely from decoupling to the present, we would expect neutrinos to be left handed and antineutrinos right handed. However, two effects modify this conclusion.

The first is that, as the neutrino trajectory is bent gravitationally by density fluctuations in the Universe, the deflection of its spin vector lags behind that of its momentum vector; gravitational fields do not conserve neutrino helicity [3-7]. Second, neutrinos of finite mass are expected to have a nonzero magnetic moment [8-16], so that propagation in galactic and cosmic magnetic fields

Published by the American Physical Society under the terms of the Creative Commons Attribution 4.0 International license. Further distribution of this work must maintain attribution to the author(s) and the published article's title, journal citation, and DOI. Funded by SCOAP ${ }^{3}$.
$[17,18]$ rotates their spins with respect to their momenta, again allowing neutrinos and antineutrinos to have an amplitude to be flipped in helicity, as first noted in Ref. [9]. Neutrinos are potential probes of cosmic and galactic magnetic fields as well as density fluctuations in the expanding Universe.

We explore here consequences of large neutrino magnetic moments on the time evolution of primordial neutrino helicities. Helicity modification by slowly varying astrophysical magnetic fields occurs via diagonal magnetic moments and is thus limited to Dirac neutrinos. In contrast, both Dirac and Majorana helicities are modified by gravitational fields [4]. The recent XENON1T report of an excess of low-energy electron events [19] has triggered interest in the possibility that a large magnetic moment of solar neutrinos, of order $\sim 1.4-2.9 \times 10^{-11} \mu_{B}\left(\equiv \mu_{1 T}\right)$, where $\mu_{B}$ is the Bohr magneton, could account for these excess events [20,21]. While beyond-the-standard-model physics, which is required for large magnetic moments, generally favors sizeable moments for Majorana rather than Dirac neutrinos [13,14,20,21], the XENON1T data, which do not distinguish diagonal from transition moments, can accommodate both neutrino types. We do not assess here the possibility of moments exceeding estimated theoretical bounds [13,22,23].

We find that even a moment several orders of magnitude smaller than $\mu_{1 T}$ could lead to significant helicity changes as Dirac neutrinos propagate through the cosmos, as well as the Milky Way. As we discuss, detection rates for primordial neutrinos are sensitive to both their helicity structure as well as whether they are Dirac or Majorana fermions. We assume $\hbar=c=1$ throughout.

We first briefly recall some properties of primordial neutrinos from standard cosmology. At temperatures $T$, small compared with the muon mass but well above $1 \mathrm{MeV}$, muons 
and tau are frozen out and the only charged leptons present are electrons and positrons; neutrinos are held in thermal equilibrium with the ambient plasma through neutral and charged current interactions. As estimated in Ref. [15], $\nu_{\tau}$ and $\nu_{\mu}$ freeze out at temperature $T_{\mu} \sim 1.5 \mathrm{MeV}$, while $\nu_{e}$ freeze out at $T_{e} \sim 1.3 \mathrm{MeV}$. However, the temperature differences at freeze-out do not effect the present temperature, $T_{\nu 0}=1.945 \pm 0.001 \mathrm{~K}=(1.676 \pm 0.001) \times 10^{-4} \mathrm{eV}$, of the various neutrino species.

The observation of neutrino oscillations establishes well that neutrino flavor eigenstates $\alpha$ are linear superpositions of mass eigenstates $i$ with Pontecorvo-Maki-NakagawaSakata flavor-mass mixing matrix elements $U_{\alpha i}$ [24]. While neutrinos decouple in flavor eigenstates, the velocity dispersion, $\delta v=\frac{1}{2} \Delta m^{2} / p^{2}$, among different mass components of momentum $p$ soon separates a given flavor state into three effectively decoherent wave packets of mass eigenstates [25]. The distribution of the present momenta $p_{0}$ of primordial neutrinos of each mass state $\left|\nu_{i}\right\rangle$ is

$$
f\left(p_{0}\right)=\frac{1}{e^{p_{0} / T_{\nu 0}}+1},
$$

independent of the neutrino mass, with total number density $n=3 \eta(3) T_{\nu 0}^{3} / 2 \pi^{2}=56.25 \mathrm{~cm}^{-3}$.

The magnetic moment of a nonzero mass Dirac neutrino is estimated in the (extended) standard model to be [8-10]

$$
\mu_{\nu}^{\mathrm{SM}} \simeq \frac{3 G_{F}}{4 \sqrt{2} \pi^{2}} m_{\nu} m_{e} \mu_{B} \simeq 3 \times 10^{-21} m_{-2} \mu_{B}
$$

$\mu_{B}=1.40 \mathrm{MHz} / \mathrm{G}$ is the Bohr magneton, and $m_{-2}$ is the neutrino mass in units of $10^{-2} \mathrm{eV}$. Diagonal moments of Majorana neutrinos must vanish, although transition moments connecting different mass eigenstates are nonzero [16]. Magnetic moments could be substantially larger than Eq. (2) predicts. According to the most recent Review of Particle Physics [26], the most sensitive upper bounds for $\mu_{\nu}$ are given by the GEMMA and Borexino experiments. The GEMMA reactor experiment [27] gives an upper limit $\mu_{\nu}<2.9 \times 10^{-11} \mu_{B}$, and Borexino [28] reports upper bounds from solar neutrinos $\mu_{\nu_{e}}<2.8 \times 10^{-11} \mu_{B}$. These bounds are comparable to the moment $\mu_{1 T}$ that could explain the XENON1T low-energy electron-event excess, which does not distinguish diagonal from transition magnetic moments.

As a neutrino with a magnetic moment propagates through magnetic fields its spin precesses (see, e.g., [29]). To set the scale, we first neglect relativistic effects; then, since the neutrino magnetic moment vector is $\mu_{B} \hat{S}=2 \mu_{B} \vec{S}$, the rotation rate of the spin is $\omega_{s}=2 \mu_{\nu} B$, where $B$ is a characteristic field strength. For example, for $B \sim 10^{-12} \mathrm{G}$, of order present intergalactic magnetic fields, the rotation rate with (2) becomes $\omega_{s} \sim 8 \times 10^{-27} m_{-2} \mathrm{~Hz}$.
Over the total age of the Universe, $t_{0} \sim 4.3 \times 10^{17} \mathrm{~s}$, the spin would rotate by a net angle $2 \mu_{\nu} B t_{0} \sim 4 \times 10^{-9} m_{-2}$ and, more generally, $\sim 10^{12}\left(\mu_{\nu} / \mu_{B}\right)\left(\mathrm{B} / 10^{-12} \mathrm{G}\right)$. Owing, however, to magnetic fields being considerably larger in the early Universe, this result underestimates the spin rotation. Transition moments do not lead to such spin rotation, and thus Majorana neutrinos would not be affected [30,31].

We calculate the neutrino spin $\vec{S}$ and its rotation in the neutrino rest frame, measuring transverse and longitudinal spin components $S_{\perp}$ and $S_{\|}$with respect to the axis of the neutrino "lab" momentum, where the lab frame is that of the "fixed stars." For rotation from an initial helicity state, for which $S_{\perp}=0$, by angle $\theta$, one has $\left|\vec{S}_{\perp}\right| /|\vec{S}|=\sin \theta$. The helicity changes from \pm 1 to $\pm \cos \theta$, and the probability of observing the helicity flipped is then $P_{f}=\sin ^{2}(\theta / 2)$; for $\theta \ll 1, P_{f} \simeq \theta^{2} / 4$.

The spin precesses in its rest frame according to

$$
\frac{d \vec{S}}{d \tau}=2 \mu_{\nu} \vec{S} \times \vec{B}_{R}
$$

where $\tau$ is the neutrino proper time, and $\vec{B}_{R}$ is the magnetic field in the rest frame.

In terms of the lab frame magnetic field and time $t$, the equations of motion of the rest frame spin are [32]

$$
\begin{gathered}
\frac{d \vec{S}_{\perp}}{d t}=2 \mu_{\nu}\left(\vec{S}_{\|} \times \vec{B}_{\perp}+\frac{1}{\gamma} \vec{S}_{\perp} \times \vec{B}_{\|}\right), \\
\frac{d S_{\|}}{d t}=2 \mu_{\nu}(\vec{S} \times \vec{B})_{\|},
\end{gathered}
$$

since in the absence of an electric field in the lab frame, $B_{\| R}=B_{\|}, B_{\perp R}=\gamma B_{\perp}$, and $d \tau=d t / \gamma$, where $\gamma=E_{\nu} / m_{\nu}$. We neglect the $\nu_{e}-e$ matter effect [33], important only for very dense matter or vanishingly small $\mu_{\nu}$.

For small deviations, $\left|S_{\perp}\right| \ll|S|$, from a pure helicity state, the $\vec{S}_{\perp} \times \vec{B}_{\|}$term in Eq. (4) is negligible; thus a neutrino of velocity $\vec{v}$ and helicity \pm 1 experiences a cumulative spin rotation with respect to its momentum,

$$
\frac{\vec{S}_{\perp}}{|\vec{S}|}= \pm 2 \mu_{\nu} \int d t \hat{v} \times \vec{B}(t)
$$

One of the larger magnetic fields a relic neutrino encounters en route to local detectors is that of our Galaxy, $B_{g} \sim 10 \mu \mathrm{G}$. Galactic fields do not point in a uniform direction, but rather change orientation over a coherence length $\Lambda_{g}$ of order kpc [34-37]. The spin orientation undergoes a random walk through the changing directions of $\vec{B}$, reducing the net rotation by a factor $\sim \sqrt{\ell_{g} / \Lambda_{g}}$, where $\ell_{g}$ is the mean crossing distance of the galaxy, of order the galactic volume $V_{g}$ divided by $\sigma_{g}$, 
its cross-sectional area. Thus the mean square spin rotation of a neutrino passing through a galaxy $(g)$ is

$$
\left\langle\theta^{2}\right\rangle_{g} \simeq\left(2 \mu_{\nu} B_{g} \frac{\Lambda_{g}}{v}\right)^{2} \frac{\ell_{g}}{\Lambda_{g}} .
$$

All quantities (except $\mu_{\nu}$ ) nominally depend on the epoch $t$. The spin rotation is larger for more massive neutrinos since $1 / v^{2}=1+m_{\nu}^{2} / p^{2}$, with $p$ the neutrino momentum.

The spin rotation for nonrelativistic neutrinos $\left(m_{\nu} \gg p \simeq T_{\nu 0}\right)$, evaluated with parameters characteristic of the Milky Way, $B_{g} \sim 10 \mu \mathrm{G}, \ell_{g} \sim 16 \mathrm{kpc}, \Lambda_{g} \sim \mathrm{kpc}$, is

$$
\left\langle\theta^{2}\right\rangle_{\mathrm{MW}} \sim 4 \times 10^{29} m_{-2}^{2}\left(\frac{\Lambda_{g}}{1 \mathrm{kpc}}\right)\left(\frac{B_{g}}{10 \mu \mathrm{G}}\right)^{2}\left(\frac{\mu_{\nu}}{\mu_{B}}\right)^{2} .
$$

A moment $\sim 1.5 \times 10^{-15} \mu_{B}$, a factor $10^{-4}$ smaller than what would account for the XENON1T excess, would yield a helicity-flip probability $P_{f}$ of order unity for $m_{-2}\left(B_{g} / 10 \mu \mathrm{G}\right)\left(\Lambda_{g} / 1 \mathrm{kpc}\right)^{1 / 2}$ itself of order unity.

Neutrinos propagate past distant galaxies before reaching the Milky Way. The effective number of galaxies a neutrino sees per unit path length is $\sim n_{g} \sigma_{g}$, where $n_{g}$ is the number density of galaxies. Integrated over the neutrino trajectory from early galaxies to now, the effective number $N_{\text {eff }}$ of galaxies a neutrino passes through is $\sim n_{g} \sigma_{g} R_{u} \sim n_{g} V_{g}\left(R_{u} / \ell_{g}\right)$, where $R_{u}$ is the present radius of the Universe. Since $n_{g} V_{g} \sim 10^{-6}$ and $R_{u} / \ell_{g} \sim 10^{6}$, a neutrino would pass through $N_{\text {eff }}$ of order unity before reaching the Milky Way. The cumulative rotation of a neutrino prior to reaching our Galaxy is comparable to the spin rotation it would undergo within the Milky Way.

We now estimate the net rotation a relic neutrino experiences from cosmic magnetic fields in the expanding Universe, from decoupling to now. We work in the metric $d s^{2}=-a(u)^{2}\left(d u^{2}-d \vec{x}^{2}\right)$, where $\vec{x}$ are the comoving spatial coordinates, and $a(u)$ is the increasing scale factor of the Universe (with $a=1$ at present); the conformal time $u$ is related to the coordinate time by $d t=a(u) d u$. Over the evolution of the Universe from decoupling, where $a\left(t_{d}\right) \equiv a_{d} \sim 10^{-10}$, to now, the cosmic magnetic field decreases; assuming that the field lines move with the overall expansion, flux conservation implies that globally $B a^{2}$ should remain essentially constant in time. As with galaxies, the coherence length $\Lambda$ of the cosmic magnetic field is not well determined, but expected to be on Mpc scales [38-40]; the coherence length reduces the net spin rotation by a factor $\sim \sqrt{\Lambda / R_{u}}$.

In order of magnitude, the ratio of the helicity-flip probability from the present cosmic field to that from a galactic field is

$$
\frac{\left\langle\theta^{2}\right\rangle_{\text {galaxy }}}{\left\langle\theta^{2}\right\rangle_{\text {cosmic }}} \sim\left(\frac{B_{g}}{B_{u}}\right)^{2} \frac{\ell_{g} \Lambda_{g}}{R_{u} \Lambda} .
$$

The magnetic field ratio is of order of at least micro- vs picogauss, while the ratio of length scales is of order $(\mathrm{kpc})^{2} /(\mathrm{Gpc} \mathrm{Mpc}) \sim 10^{-9}$, which would indicate a scale of neutrino spin rotation in galaxies up to 3 orders of magnitude larger than in cosmic magnetic fields. However, in assessing whether cosmic rotation is competitive with the rotation from the galactic magnetic field, it is necessary, in addition to determining better the cosmic and galactic magnetic fields and correlation lengths, to take into account the larger cosmic fields as well as smaller coherence lengths at earlier times.

We turn now to this latter task. We start from the squared rotation in Eq. (6), written in terms of $u$ for relativistic neutrinos, with $c$ denoting "cosmic,"

$$
\left\langle\theta^{2}\right\rangle_{c}=4 \mu_{\nu}^{2}\left\langle\left[\int d u a(u) \vec{B}_{\perp}(u)\right]^{2}\right\rangle_{c}
$$

with the expectation value in the cosmic background. The correlation function of the cosmic magnetic field, in an otherwise isotropic background, has the structure

$$
\left\langle B_{i}(\vec{x}) B_{j}\left(\vec{x}^{\prime}\right)\right\rangle=\left(-\delta_{i j} \nabla^{2}+\nabla_{i} \nabla_{j}\right) F(r)+\epsilon_{i j k} \nabla_{k} G(r),
$$

where $r=\left|\vec{x}-\vec{x}^{\prime}\right|$, and $F$ is the normal and $G$ the helical field [41] correlation. The latter does not contribute to the spin rotation since $\nabla_{z} G(r)$ is odd in $\vec{x}-\vec{x}^{\prime}$, and thus its contribution for transverse spin components $\sim \int d u d u^{\prime} \partial_{z} G(r)$ vanishes by symmetry.

The normal correlation has the Fourier structure [42]

$\left\langle B_{i}(\vec{x}) B_{j}\left(\vec{x}^{\prime}\right)\right\rangle=\int \frac{d^{3} k}{(2 \pi)^{3}} \frac{\delta_{i j}-\hat{k}_{i} \hat{k}_{j}}{2} P_{B}(k) e^{i \vec{k} \cdot\left(\vec{x}-\vec{x}^{\prime}\right)}$,

where, in another convention for the correlation function [18], $P_{B}(k)=(2 \pi)^{2} E_{M}(k) / k^{2}$. Equation (12) implies that

$$
\left\langle\vec{B}^{2}\right\rangle=\int \frac{d^{3} k}{(2 \pi)^{3}} P_{B}(k) .
$$

The schematic structure of $P_{B}$ is a power law $\sim \alpha k^{s}$ at small $k$ out to a wave vector $k_{*}$ (called $k_{i}$ in Ref. [18]), followed by a sharper falloff, $\sim \beta k^{-q}$ beyond $k_{*}$, with $q>3$ and $\beta=\alpha k_{*}^{s+q}$. The sign of $s$ is uncertain $[18,43]$, but infrared convergence of the integral in Eq. (15) requires $s>-2$. With this approximate form, Eq. (13) implies $\alpha \simeq 2 \pi^{2}(s+3)(q-3)\left\langle\vec{B}^{2}\right\rangle_{c} /(s+q) k_{*}^{s+3}$.

With Eq. (12) and taking the $z$ axis along the neutrino velocity, Eq. (10) becomes 


$$
\begin{aligned}
\left\langle\theta^{2}\right\rangle_{c}= & 4 \mu_{\nu}^{2} \int d u d u^{\prime} a(u) a\left(u^{\prime}\right) \\
& \times \int \frac{d^{3} k}{(2 \pi)^{3}} e^{i k_{z}\left(u-u^{\prime}\right)} \frac{1-k_{z}^{2} / k^{2}}{2} P_{B}(k) .
\end{aligned}
$$

Since the scale of $k$ is $\gg 1 / u$, the $u$ integrals are vanishingly small except in the neighborhood of $k_{z}=0$, and to a first approximation we set $k_{z}=0$ in $\left(1-k_{z}^{2} / k^{2}\right) P_{B}(k)$. Then the $k_{z}$ integral gives a factor $2 \pi \delta\left(u-u^{\prime}\right)$, and

$$
\left\langle\theta^{2}\right\rangle_{c} \simeq \frac{\mu_{\nu}^{2}}{\pi} \int_{u_{d}}^{u_{0}} d u a(u)^{2} \int_{0}^{\infty} d k_{\perp} k_{\perp} P_{B}\left(k_{\perp}\right),
$$

where 0 denotes present values, $u_{0}=3 t_{0}$, and $d$ denotes neutrino decoupling. Here,

$$
\int_{0}^{\infty} d k_{\perp} k_{\perp} P_{B}\left(k_{\perp}\right) \simeq 2 \pi^{2} \eta \frac{\left\langle\vec{B}^{2}\right\rangle}{k_{*}} .
$$

With conservation of flux, $\left\langle\vec{B}^{2}(u)\right\rangle \simeq B_{0}^{2} / a(u)^{4}$, and $k_{*}(u) \sim 2 \pi / \Lambda_{0} a(u)^{1 / 2}$ [18]. The factor $\eta=(s+3)(q-3) /$ $(s+2)(q-2)$ is not strongly dependent on the spectral indices, and for simplicity we take $\eta=1 / 2$ (corresponding to $s=2$ and $q=2+5 / 3)$. Then,

$$
\left\langle\theta^{2}\right\rangle_{c}=\frac{1}{2} \mu_{\nu}^{2} B_{0}^{2} \Lambda_{0} \int_{u_{d}}^{u_{0}} \frac{d u}{a(u)^{3 / 2}} .
$$

The main contribution to the integral is from the radiation-dominated era, from the time of neutrino decoupling $u_{d}$ to the time of matter-radiation equality $u_{\text {eq }}$, where $a\left(t_{\mathrm{eq}}\right) \equiv a_{\mathrm{eq}} \sim 0.8 \times 10^{-4}$. In this era $a \propto u$, and

$$
\int_{u_{d}}^{u_{\mathrm{eq}}} \frac{d u}{a(u)^{3 / 2}} \simeq \frac{2 u_{0}}{a_{\mathrm{eq}}^{1 / 2} a_{d}^{1 / 2}} \simeq 2 \times 10^{7} u_{0},
$$

since $u_{\mathrm{eq}}$ is related to $u_{0}$ by $u \propto a^{1 / 2}$ in the matterdominated era. By comparison, in the matter-dominated era,

$$
\int_{u_{\mathrm{eq}}}^{u_{0}} \frac{d u}{a(u)^{3 / 2}} \simeq \frac{u_{0}}{2 a_{\mathrm{eq}}},
$$

a factor $\sqrt{a_{d} / a_{\mathrm{eq}}} / 4 \sim 10^{-4}$ smaller.

Altogether,

$$
\begin{aligned}
\left\langle\theta^{2}\right\rangle_{c} & \simeq 9\left(\frac{\Lambda_{0}}{R_{u}}\right) \frac{\left(\mu_{\nu} t_{0} B_{0}\right)^{2}}{a_{\mathrm{eq}}^{1 / 2} a_{d}^{1 / 2}} \\
& \simeq 2 \times 10^{27}\left(\frac{\Lambda_{0}}{1 \mathrm{Mpc}}\right)\left(\frac{B_{0}}{10^{-12} \mathrm{G}}\right)^{2}\left(\frac{\mu_{\nu}}{\mu_{B}}\right)^{2},
\end{aligned}
$$

independent of the neutrino momentum. To within uncertainties in magnetic fields, correlation lengths, and neutrino masses, the estimated spin rotation in the cosmos is basically comparable to that in galaxies.

If the low-energy electron-event excess found in the XENON1T experiment [19] does arise from a neutrino magnetic moment, and the neutrino is a Dirac particle, its diagonal moment could lead to a significant spin rotation. A magnetic moment of order $10^{-2} \mu_{1 T}$ would still produce a spin rotation in the range of detectability. On the other hand, if the neutrino is a Majorana particle, the excess would occur entirely from transition magnetic moments, and magnetic fields would produce no helicity changes.

Having described the expected spin rotation of relic neutrinos, we turn to their detection. The most promising approach is to capture neutrinos on beta unstable nuclear targets. Particularly favorable for detecting primordial neutrinos is the inverse tritium beta decay (ITBD) [1,3], $\nu_{e}+{ }^{3} \mathrm{H} \rightarrow{ }^{3} \mathrm{He}+e^{-}$, the reaction inverse to tritium beta decay, ${ }^{3} \mathrm{H} \rightarrow{ }^{3} \mathrm{He}+e^{-}+\bar{\nu}_{e}$. The ITBD would yield a distinct signature of a monoenergetic peak separated from the end point of the tritium beta decay by $2 m_{\nu}$.

The cross section for capture of a neutrino in mass state $i$ on tritium is [3]

$$
\begin{aligned}
& \sigma_{i}^{h}\left(p, p_{e}\right) \\
& =\frac{G_{F}^{2}}{2 \pi v_{i}}\left|V_{\mathrm{ud}}\right|^{2}\left|U_{e i}\right|^{2} F\left(Z, E_{e}\right) \frac{m\left({ }^{3} \mathrm{He}\right)}{m\left({ }^{3} \mathrm{H}\right)} E_{e} p_{e} A_{i}^{h}\left(\bar{f}^{2}+3 \bar{g}^{2}\right),
\end{aligned}
$$

with $V_{\text {ud }}$ the up-down quark element of the CabibboKobayashi-Maskawa matrix, the $U_{e i}$ are the neutrino mixing matrix elements, and $F\left(Z, E_{e}\right)$ is the Fermi Coulomb correction for the electron- ${ }^{3} \mathrm{He}$ system. The $\bar{f}$ and $\bar{g}$ are the nuclear form factors for Fermi and GamowTeller transitions, and the neutrino helicity-dependent factor is $A_{i}^{ \pm}=1 \mp \beta_{i}$, where $\beta_{i}=v_{i} / c$.

The total ITBD rate is given by $\sigma_{i}^{h} v_{i}$ integrated over the distribution (1) of neutrinos and summed over mass states $i$. For Dirac neutrinos with spin rotated by $\theta_{i}$, both negative and positive helicity states, weighted by $\frac{1}{2}\left(1 \pm \cos \theta_{i}\right)$, contribute and yield the neutrino dependence in the rate

$$
A_{\mathrm{eff}, D}=\sum_{i, h= \pm}\left|U_{e i}\right|^{2}\left\langle A_{i}^{h}\right\rangle_{T}=1+\sum_{i}\left|U_{e i}\right|^{2}\left\langle\beta_{i} \cos \theta_{i}\right\rangle_{T} .
$$

The subscript $T$ includes the thermal average over the distribution (1) as well as the average of the spin rotation over the neutrino's history.

Majorana neutrinos, as noted, have no diagonal magnetic moments and cannot flip spin in a slowly varying magnetic field, so that $\langle\cos \theta\rangle=1$. Since the ITBD measures both Majorana neutrinos and antineutrinos, 


$$
\begin{aligned}
A_{\mathrm{eff}, M}= & \left(1+\sum_{i}\left|U_{e i}\right|^{2}\left\langle\beta_{i}\right\rangle_{T}\right) \\
& +\left(1-\sum_{i}\left|U_{e i}\right|^{2}\left\langle\beta_{i}\right\rangle_{T}\right)=2,
\end{aligned}
$$

independent of the neutrino masses and spin rotation by cosmic gravitational fluctuations [4].

Figure 1 shows the $A_{\text {eff }}$ as a function of the mass of the lightest neutrino, for both Dirac and Majorana neutrinos with normal and inverted mass hierarchies. For neutrinos maintaining their original helicity $\left(\theta_{i}=0\right)$, the $A_{\text {eff }}$ are the solid curves. As the mass of the lightest neutrino approaches zero, $A_{\text {eff, } D}$ approaches $1+\left|U_{e 1}\right|^{2}=1.6794$ in the normal and $1+\left|U_{e 3}\right|^{2}=1.0216$ in the inverted hierarchy. When the lightest neutrino mass rises and all neutrinos become nonrelativistic, $\quad A_{\text {eff }, D}=1+\left(7 \pi^{4} T_{\nu 0} / 180 \zeta(3)\right) \sum_{i}\left|U_{e i}\right|^{2} / m_{i}$ eventually approaches unity independent of the mass hierarchy; $A_{\text {eff }}$ is always larger for Majorana than Dirac neutrinos, independent of the mass hierarchy and the mass of the lightest neutrino.

The dashed curves in Fig. 1 show the dependence of $A_{\text {eff, } D}$ on the lightest neutrino mass for complete helicity flip, $\theta_{i}=\pi$. For partial spin rotation, $A_{\mathrm{eff}, D}$ lies between the

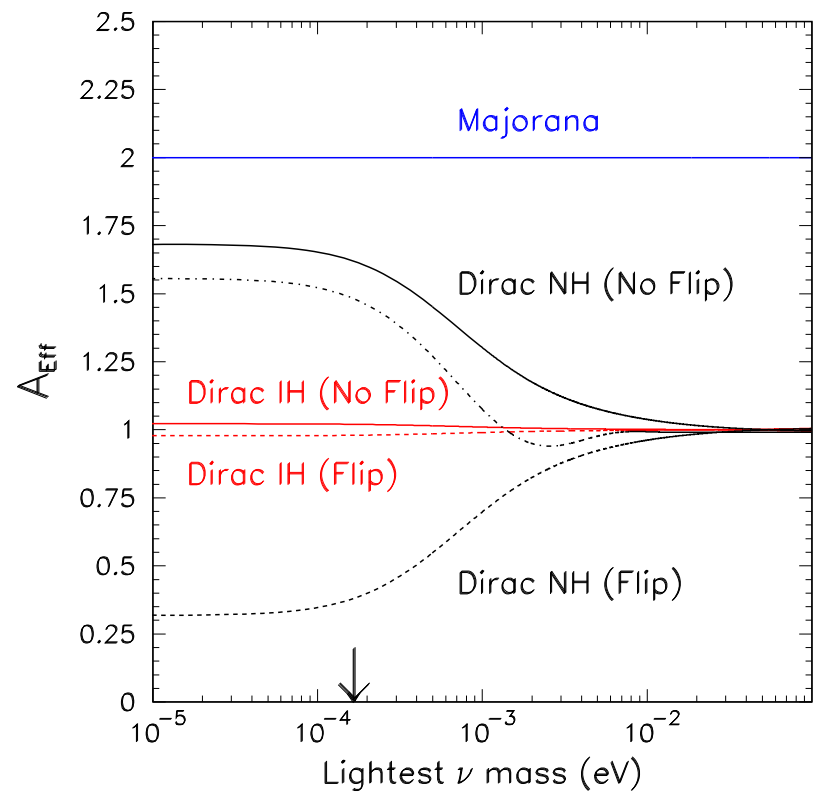

FIG. 1. The coefficient $A_{\text {eff }}$ vs mass of the lightest neutrino for Dirac and Majorana neutrinos, for the normal $(\mathrm{NH})$ and inverted (IH) hierarchies. The dashed curves show the extreme case of complete helicity flip from left to right handed. The dash-dotted curve shows the result for Dirac NH neutrinos with $\left\langle\theta^{2}\right\rangle$ given by the Milky Way estimate (7), with $B=10 \mu \mathrm{G}, \Lambda_{g}=1 \mathrm{kpc}$, and $\mu_{\nu}=5 \times 10^{-14} \mu_{B}$. The present neutrino temperature $T_{\nu 0}$ (arrow) demarcates the transition of the lightest neutrino from relativistic to nonrelativistic. solid and dashed curves. When $\theta_{i}=\pi / 2$, the amplitudes to be left and right handed are equal and $A_{\text {eff, } D}=1$. To illustrate the qualitative dependence of the helicity-flip probability on $\mu_{\nu}$ in Fig. 1, we show $A_{\text {eff, } D}$ for Dirac neutrinos passing through the Milky Way as the dashdotted curve, calculated from Eq. (7) for small angle bending with $B_{g}=10 \mu \mathrm{G}$ and $\Lambda_{g}=1 \mathrm{kpc}$, and with $\mu_{\nu}=5 \times 10^{-14} \mu_{B}, 2$ orders of magnitude smaller than the magnetic moment XENON1T would need to explain their event excess. The value of $\mu_{\nu}=5 \times 10^{-14} \mu_{B}$ is also below the upper bound derived from the analysis of solar neutrino data $[44,45]$ and is consistent with the upper bound deduced from the stellar energy loss [46]. If the magnetic moment of normal hierarchy Dirac neutrinos is of order of that suggested by XENON1T, then for the characteristic parameters assumed for cosmic or galactic magnetic fields the neutrino spin rotations would no longer be small; the mean $\cos \theta$ would decrease $A_{\text {eff, } D}$ to essentially unity, with a concomitant decrease in the ITBD detection rate. A magnetic moment of the standard model prediction of Eq. (2) would affect $A_{\text {eff, } D}$ insignificantly. In contrast, a value of $\mu_{\nu}=10^{-14} \mu_{B}$, the naturalness upper bound obtained from an EFT analysis [13,14], would have a significant effect on $A_{\text {eff }, D}$.

Figure 1 illustrates how measurements of the rate of relic neutrinos can distinguish Dirac from Majorana neutrinos, with an accuracy that will improve as knowledge of the correct hierarchy as well as the lightest mass come into sharper focus. As the dash-dotted curve indicates, the interesting regime is of bending not too small to be indistinguishable and not so large that all spins have comparable probability of being left and right handed. This regime is characterized by a falloff in $A_{\text {eff, } D}$ and the ITBD detection rate with increasing light neutrino mass. Unfortunately, it becomes increasingly difficult to resolve the relic neutrino events from the tritium beta decay background for smaller neutrino mass. Inventing novel techniques to probe the region of interest shown in Fig. 1 remains a challenge.

In conclusion, investigating the implications of a possible large neutrino magnetic moment beyond that in the standard model on the helicities of relic neutrinos as they propagate through the cosmic and galactic magnetic fields, we find significant helicity modifications, even if $\mu_{\nu}$ is 2 orders of magnitude smaller than that suggested by the XENON1T result. The present estimates of neutrino spin rotation can be sharpened by using detailed maps as well as numerical simulations of the astrophysical magnetic fields, e.g., [47-50]. In addition, the spin rotation of $\mathrm{MeV}$ energy neutrinos from the diffuse supernova background [51] as well as from neutron stars [9] is also potentially detectable, although using different experimental techniques than for relic neutrinos (e.g., with the Gd-doped Super-K detector and the inverse beta decay reaction) [52]. 
This research was supported in part by NSF Grant No. PHY18-22502. We thank Michael Turner and Tanmay Vachaspati for helpful discussions.

[1] S. Weinberg, Universal neutrino degeneracy, Phys. Rev. 128, 1457 (1962).

[2] S. Betts et al., Development of a relic neutrino detection experiment at PTOLEMY: Princeton Tritium Observatory for Light, Early-Universe, Massive-Neutrino Yield (Community Summer Study 2013: Snowmass on the Mississippi), arXiv:1307.4738; E. Baracchini et al., PTOLEMY: A proposal for thermal relic detection of massive neutrinos and directional detection of $\mathrm{MeV}$ dark matter, arXiv:1808.01892.

[3] A. J. Long, C. Lunardini, and E. Sabancilar, Detecting nonrelativistic cosmic neutrinos by capture on tritium: Phenomenology and physics potential, J. Cosmol. Astropart. Phys. 08 (2014) 038.

[4] G. Baym and J. C. Peng, Evolution of primordial neutrino helicities incosmic gravitational inhomogeneities, arXiv: 2103.11209.

[5] G. Duda, G. Gelmini, and S. Nussinov, Expected signals in relic neutrino detectors, Phys. Rev. D 64, 122001 (2001).

[6] A. J. Silenko and O. V. Teryaev, Semiclassical limit for Dirac particles interacting with a gravitational field, Phys. Rev. D 71, 064016 (2005).

[7] M. Dvornikov, Neutrino spin oscillations in gravitational fields, Int. J. Mod. Phys. D 15, 1017 (2006); Spin effects in neutrino gravitational scattering, arXiv:1911.08317.

[8] W. J. Marciano and A. I. Sanda, Exotic decays of the muon and heavy leptons in gauge theories, Phys. Lett. 67B, 303 (1977).

[9] K. Fujikawa and R. Shrock, The Magnetic Moment of a Massive Neutrino and Neutrino Spin Rotation, Phys. Rev. Lett. 45, 963 (1980).

[10] B. W. Lee and R.E. Shrock, Natural suppression of symmetry violation in gauge theories: Muon- and electron-leptonnumber nonconservation, Phys. Rev. D 16, 1444 (1977).

[11] B. W. Lynn, Magnetic moment of massive neutrinos and the cosmic helium abundances, Phys. Rev. D 23, 2151 (1981).

[12] S. L. Shapiro and I. Wasserman, Massive neutrinos, helium production and the primordial magnetic field, Nature (London) 289, 657 (1981).

[13] N. F. Bell, V. Cirigliano, M. J. Ramsey-Musolf, P. Vogel, and M. B. Wise, How Magnetic is the Dirac Neutrino? Phys. Rev. Lett. 95, 151802 (2005).

[14] N. F. Bell, M. Gorchtein, M. J. Ramsey-Musolf, P. Vogel, and P. Wang, Model independent bounds on magnetic moments of Majorana neutrinos, Phys. Lett. B 642, 377 (2006).

[15] A. D. Dolgov, Neutrinos in cosmology, Phys. Rep. 370, 333 (2002).

[16] C. Giunti and A. Studenikin, Neutrino electromagnetic interactions: A window to new physics, Rev. Mod. Phys. 87, 531 (2015).

[17] K. Subramanian, The origin, evolution and signatures of primordial magnetic fields, Rep. Prog. Phys. 79, 076901 (2016).

[18] T. Vachaspati, Progress on cosmological magnetic fields, arXiv:2010.10525.
[19] E. Aprile et al. (XENON1T Collaboration), Excess electronic recoil events in XENON1T, Phys. Rev. D 102, 072004 (2020).

[20] O. G. Miranda, D. K. Papoulias, M. Tortola, and J. W. F. Valle, XENON1T signal from transition neutrino magnetic moments, Phys. Lett. B 808, 135685 (2020).

[21] K. S. Babu, S. Jana, and M. Lindner, Large neutrino magnetic moments in the light of recent experiments, J. High Energy Phys. 10 (2020) 040.

[22] K. Enqvist, P. Olesen, and V. Semikoz, Galactic Dynamo and Nucleosynthesis Limit on the Dirac Neutrino Masses, Phys. Rev. Lett. 69, 2157 (1992).

[23] V. B. Semikoz, Cosmological bounds on neutrino degeneracy and the dirac neutrino magnetic moment, Phys. Part. Nucl. 48, 1021 (2017).

[24] I. Esteban, M. C. Gonzalez-Garcia, M. Maltoni, I. MartinezSoler, and T. Schwetz, Updated fit to three neutrino mixing: Exploring the accelerator-reactor complementarity, J. High Energy Phys. 01 (2017) 087.

[25] The best-value fits to the mass squared differences, $\Delta m_{i j}^{2} \equiv m_{i}^{2}-m_{j}^{2}$, are [24] $\Delta m_{21}^{2}=(7.50+0.19-0.17) \times 10^{-5}$, $\Delta m_{31, N}^{2}=(2.524+0.039-0.040) \times 10^{-3}$, and $\Delta m_{31, I}^{2}=$ $(-2.514+0.038-0.041) \times 10^{-3} \mathrm{eV}^{2}$.

[26] P. A. Zyla et al. (Particle Data Group), Review of particle physics, Prog. Theor. Exp. Phys. (2020), 083C01.

[27] A. G. Beda, V. B. Brudanin, V. G. Egorov, D. V. Medvedev, V.S. Pogosov, E. A. Shevchik, M. V. Shirchenko, A.S. Starostin, and I. V. Zhitnikov, (Gemma experiment): The results of neutrino magnetic moment search, Phys. Part. Nucl. Lett. 10, 139 (2013).

[28] M. Agostini et al. (Borexino Collaboration), Limiting neutrino magnetic moments with Borexino phase-II solar neutrino data, Phys. Rev. D 96, 091103 (2017).

[29] V. B. Semikoz, Neutrino spin kinetics in a medium with magnetic field, Phys. Rev. D 48, 5264 (1993).

[30] V. B. Semikoz, Can we distinguish Majorana and Dirac neutrinos in solar neutrino experiments? Nucl. Phys. B498, 39 (1997).

[31] J. Barranco, D. Delepine, V. Gonzalez-Macias, C. LujanPeschard, and M. Napsucial, Scattering processes could distinguish Majorana from Dirac neutrinos, Phys. Lett. B 739, 343 (2014).

[32] V. Bargmann, L. Michel, and V. L. Telegdi, Precession of the Polarization of the Particles Moving in a Homogeneous Electromagnetic Field, Phys. Rev. Lett. 2, 435 (1959).

[33] M. B. Voloshin, M. I. Vysotsky, and L. B. Okun, Neutrino electrodynamics and possible consequences for solar neutrinos, Sov. Phys. JETP 64, 446 (1986).

[34] M. S. Turner, E. N. Parker, and T. J. Bogdan, Magnetic monopoles and the survival of galactic magnetic fields, Phys. Rev. D 26, 1296 (1982).

[35] E. G. Zweibel, Cosmic-ray history and its implications for galactic magnetic fields, Astrophys. J. 587, 625 (2003).

[36] J. L. Han, K. Ferrière, and R. N. Manchester, The spatial energy spectrum of magnetic fields in our Galaxy, Astrophys. J. 610, 820 (2004).

[37] H.-Y. K. Yang, M. Ruszkowski, and E. Zweibel, The Fermi bubbles: gamma-ray, microwave and polarization signatures of leptonic AGN jets, Mon. Not. R. Astron. Soc. 436, 2734 (2013). 
[38] R. A. Batista, A. Saveliev, G. Sigl, and T. Vachaspati, Probing intergalactic magnetic fields with simulations of electromagnetic cascades, Phys. Rev. D 94, 083005 (2016).

[39] A. Neronov, A. M. Taylor, C. Tchernin, and I. Vovk, Measuring the correlation length of intergalactic magnetic fields from observations of gamma-ray induced cascades, Astron. Astrophys. 554, A31 (2013).

[40] M. Ackermann et al. (Fermi-LAT Collaboration), The search for spatial extension in high-latitude sources detected by the Fermi large area telescope, Astrophys. J. Suppl. 237, 32 (2018).

[41] A. Brandenburg, R. Durrer, T, Kahniashvili, S. Mandalc, and W.W. Yin, Statistical properties of scale-invariant helical magnetic fields and applications to cosmology, J. Cosmol. Astropart. Phys. 08 (2018) 034.

[42] P. A. R. Ade et al. (Planck Collaboration), Planck 2015 results, XIX. Constraints on primordial magnetic fields, Astron. Astrophys. 594, A19 (2016).

[43] K.E. Kunze, Effects of helical magnetic fields on the cosmic microwave background, Phys. Rev. D 86, 103010 (2012).

[44] O. G. Miranda, T. I. Rashbar, A. I. Rez, and J. W. F. Valle, Constraining the Neutrino Magnetic Moment with Antineutrinos from the Sun, Phys. Rev. Lett. 93, 051304 (2004).

[45] O. G. Miranda, T. I. Rashbar, A. I. Rez, and J. W. F. Valle, Enhanced solar antineutrino flux in random magnetic fields, Phys. Rev. D 70, 113002 (2004).

[46] G. G. Raffelt, Limits on neutrino electromagnetic properties: An update, Phys. Rep. 320, 319 (1999).
[47] R. Banerjee and K. Jedamzik, Evolution of cosmic magnetic fields: From the very early Universe, to recombination, to the present, Phys. Rev. D 70, 123003 (2004).

[48] J. L. Han, R. N. Manchester, W. van Straten, and P. Demorest, Pulsar rotation measures and large-scale magnetic field reversals in the galactic disk, Astrophys. J. Suppl. 234, 11 (2018).

[49] J. Xu and J. L. Han, Magnetic fields in the solar vicinity and in the Galactic halo, Mon. Not. R. Astron. Soc. 486, 4275 (2019).

[50] F. Boulanger et al., IMAGINE: A comprehensive view of the interstellar medium, galactic magnetic fields and cosmic rays, J. Cosmol. Astropart. Phys. 08 (2018) 049.

[51] J. F. Beacom, The diffuse supernova neutrino background, Annu. Rev. Nucl. Part. Sci. 60, 439 (2010).

[52] Supernova and neutron star neutrinos are rotated by $\sim \mu_{\nu} B R / c$, in magnetic fields upward of $10^{12} \mathrm{G}$, with $R$ the distance over which the magnetic fields are large. For magnetic fields in neutron stars and supernovae reasonably coherent over the regime of high field, then $\theta^{2} \sim 2 \times 10^{27}(R / 10 \mathrm{~km})^{2}\left(B / 10^{12} \mathrm{G}\right)^{2}\left(\mu_{\nu} / \mu_{B}\right)^{2}$, comparable in scale to the rotation of relic neutrinos in galaxies. Characteristically, $B$ scales as $R^{-2}$ in core collapse supernovae, so that the rotation would be largest for neutrinos from neutron stars, during formation or later cooling. The preponderance of diffuse background supernova and neutron star neutrinos are produced after redshift $z_{s}$ at most of order 10; the rotation of these neutrinos in cosmic magnetic fields, given by Eq. (17) using the integral in Eq. (19) with $1 / a_{\mathrm{eq}} \rightarrow 1+z_{s}$, is negligible in comparison. 